\title{
Strong Chang's Conjecture, Semi-Stationary Reflection, the Strong Tree Property and two-cardinal square principles
}

by

\section{Víctor Torres-Pérez (Wien) and Liuzhen Wu (Beijing)}

\begin{abstract}
We prove that a strong version of Chang's Conjecture implies both the Strong Tree Property for $\omega_{2}$ and the negation of the square principle $\square(\lambda, \omega)$ for every regular cardinal $\lambda \geq \omega_{2}$.
\end{abstract}

1. Introduction. In these notes we consider two equivalent principles: a strong version of Chang's Conjecture and the Semi-Stationary Reflection Principle. Given two sets $x, y$, we write $x \sqsubseteq y$ whenever $x \subseteq y$ and $x \cap \omega_{1}=$ $y \cap \omega_{1}$.

Definition 1.1. The principle $\mathrm{CC}^{*}$ asserts that for every regular cardinal $\kappa \geq \omega_{2}$, there are arbitrary large $\theta$ such that the following statement $\mathrm{CC}(\kappa, \theta)$ holds: For every countable $M \prec H_{\theta}$ and every $a \in[\kappa]^{\omega_{1}}$, there is a countable $M^{*} \prec H_{\theta}$ and $a^{*} \in M^{*} \cap[\kappa]^{\omega_{1}}$ such that $a^{*} \supseteq a$ and $M^{*} \sqsupseteq M$.

A first generalization of Chang's Conjecture of this kind was given by Shelah (see [21, Theorem 1.3, p. 398]). Similar general versions were studied in [25] and [5]. The Semi-Stationary Reflection Principle (SSR) was introduced by Shelah [22, Chapter XIII, Definition 1.5]. Given an ordinal $\lambda$ and a set $X \subseteq[\lambda]^{\omega}$, we say $X$ is semi-stationary in $[\lambda]^{\omega}$ if its $\sqsubseteq$-upward closure is stationary, i.e. the set $\left\{y \in[\lambda]^{\omega}: \exists x \in X(x \sqsubseteq y)\right\}$ is stationary. It is clear that every stationary set is semi-stationary.

DEFinition 1.2. The principle SSR asserts that the following statement $\operatorname{SSR}(\lambda)$ holds for every ordinal $\lambda \geq \omega_{2}$ : for every semi-stationary subset $X \subseteq[\lambda]^{\omega}$, there is $W \in[\lambda]^{\omega_{1}}$ with $W \supseteq \omega_{1}$ such that $X \cap[W]^{\omega}$ is semistationary in $[W]^{\omega}$.

2010 Mathematics Subject Classification: 03E05, 03E30, 03E55.

Key words and phrases: Strong Tree Property, square principles, Semi-Stationary Reflection Principle, Chang's Conjecture, Rado's Conjecture.

Received 1 October 2015; revised 9 May 2016.

Published online 2 December 2016. 
Döbler and Schindler proved that $\mathrm{CC}^{*}$ and SSR are equivalent (see [5, Theorem 5.7]). Shelah showed that SSR is equivalent to the following statement:

$(\dagger)$ Every poset preserving stationary subsets of $\omega_{1}$ is semiproper (see [22, Chapter XIII, 1.7]). Although these principles are consequences of the Weak Reflection Principle (see for example [17]) or Rado's Conjecture [4, they have many important consequences of their own: In [9], it was already shown that $(\dagger)$ implies that the ideal $\mathrm{NS}_{\omega_{1}}$ is precipitous. It was shown that under a weaker version of $\mathrm{CC}^{*}$, the existence of a special $\aleph_{2}$-Aronszajn tree is equivalent to $\mathrm{CH}$ (see [26]), and that SSR implies the Singular Cardinal Hypothesis [19] and the negation of $\square(\lambda)$ for all regular cardinals $\lambda \geq \omega_{2}$. The present authors [28] showed recently that under a weak version of $\mathrm{CC}^{*}$, the negation of $\mathrm{CH}$ entails the Tree Property for $\omega_{2}$.

In Section 3, we discuss the relationship between $\mathrm{CC}^{*}$ and the Strong Tree Property. Looking for sufficient conditions for a tree to have a cofinal branch has led to many interesting combinatorial results. We recall that an infinite regular cardinal $\kappa$ has the Tree Property $(\mathrm{TP}(\kappa))$ if for every tree of height $\kappa$ with levels of size $<\kappa$, there is a cofinal branch. König's Lemma states that $\operatorname{TP}(\omega)$ holds [14], while Aronszajn showed that there is a tree of height $\omega_{1}$ with each level at most countable and with no cofinal branches (see [15, Theorem 6, p. 96]). Baumgartner [1] proved that the Proper Forcing Axiom PFA implies TP $\left(\omega_{2}\right)$. However, $\operatorname{TP}\left(\omega_{2}\right)$ turned out to be equiconsistent with the existence of a weakly compact cardinal ([16, Theorem 5.9] and [6]).

Jech introduced a strengthening of the Tree Property, now called the Strong Tree Property (see Section 3 for the definition). He noticed that an inaccessible cardinal $\kappa$ has the Strong Tree Property if and only if $\kappa$ is strongly compact (see [12, p. 174]). Weiß [31] showed that PFA implies $\aleph_{2}$ has the Strong Tree Property. Sakai and Veličković [19] proved that SSR, together with $\mathrm{MA}_{\omega_{1}}$ (Cohen), implies the Strong Tree Property at $\omega_{2}$.

In this note, we show that it is enough to assume SSR and $\neg \mathrm{CH}$ for $\omega_{2}$ to have the Strong Tree Property. We remark that SSR is consistent with both $\mathrm{CH}$ and $\neg \mathrm{CH}$, and that $\mathrm{CH}$ implies $\neg \mathrm{TP}\left(\omega_{2}\right)$. Therefore, our result is in certain sense optimal.

In Section 4, we study the relationship between SSR and the square principle $\square(\lambda, \omega)$ for every regular cardinal $\lambda \geq \omega_{2}$. The original square principle $\square_{\lambda}$ was introduced by Jensen [13. He showed that $\square_{\lambda}$ holds in L for every uncountable cardinal $\lambda$. Schimmerling [20] generalized this square principle to weaker versions of the form $\square_{\kappa, \lambda}$. These two-cardinal versions have been extensively studied so far. For example, after the works of Cummings-Magidor and Baumgartner, we have a complete picture of the relationship between 
MM and square principles of the form $\square_{\kappa, \lambda}$ ([2], [3]). Some partial results were also given on relations between Rado's Conjecture (RC) and $\square_{\kappa, \lambda}$ ([26], [27]). Sakai established in unpublished notes [18] a rather complete picture of relations between SSR and the square principles $\square_{\kappa, \lambda}$.

The square principle $\square(\lambda)$ (see Definition 4.1) has also been studied in several instances. Jensen showed that in $L$, if $\lambda>\omega$ is regular and $\square(\lambda)$ holds, then $\lambda$ is not weakly compact (see [13, Theorem 6.1]). It has been proven that the negation of $\square(\lambda)$ for all regular cardinal $\lambda \geq \omega_{2}$ is implied by the Proper Forcing Axiom (Todorčević [24]), the Weak Reflection Principle (Veličković [29]), Rado's Conjecture (Todorčević [25]) and more recently by SSR (Sakai-Veličković [19]). Regarding a two-cardinal version $\square(\kappa, \lambda)$ (see Definition 4.1) and its relation to other combinatorial principles, some results have been already established, for example in [23] and [27]. In this paper, we prove that SSR is enough to have the negation of $\square(\lambda, \omega)$ for every regular cardinal $\lambda \geq \omega_{2}$.

2. Preliminaries. In these notes we will consider several types of stationary sets.

Given a limit ordinal $\gamma$, a subset $A \subseteq \gamma$ is unbounded in $\gamma$ if $\sup (A)=\gamma$, and closed in $\gamma$ if for every limit ordinal $\beta<\gamma$, if $A \cap \beta$ is unbounded in $\beta$, then $\beta \in A$. A set $A \subseteq \gamma$ is often called a club set in $\gamma$ if it is closed and unbounded in $\gamma$. A set $S \subseteq \gamma$ is stationary if $S \cap A \neq \emptyset$ for every $A$ club in $\gamma$.

The following result involving stationary sets is known as Fodor's Lemma or the Pressing Down Lemma for ordinals.

Lemma 2.1 (Fodor [7]). Let $\kappa$ be a regular uncountable cardinal. Then for every stationary $S \subseteq \kappa$, and for every $f: S \rightarrow \kappa$ such that $f(\alpha)<\alpha$ for every $\alpha \in S$, there is $\xi<\kappa$ such that $f^{-1}(\{\xi\})$ is stationary.

A general version of a stationary set was given originally by Jech. We will also use an equivalent version due to Kueker (see for example [10, Theorem $8.28]$ ). Given an infinite set $A$ and a regular cardinal $\mu$, we denote by $[A]^{<\mu}$ the collection of subsets of $A$ of size $<\mu$. Similarly, let $[A]^{\mu}$ denote the collection of all subsets of $A$ of size $\mu$. We say that a set $S \subseteq[A]^{\omega}$ is stationary in $[A]^{\omega}$ if for every function $F:[A]^{<\omega} \rightarrow A$, there is $X \in S$ such that $F(e) \in X$ for every $e \in[X]^{<\omega}$.

The following lemma is a generalized version of the Pressing Down Lemma (see [10, Theorem 8.24]).

Lemma $2.2(\mathrm{Jech})$. For every stationary set $S \subseteq[A]^{\omega}$ and every $f: S \rightarrow A$ such that $f(X) \in X$ for every $X \in S$, there is a $\in A$ such that $f^{-1}(\{a\})$ is stationary. 
In general, we say that a set $S \subseteq[A]^{\mu}$ is weakly stationary if for every $F:[A]^{<\omega} \rightarrow A$, there is $X \in S$ such that $F(e) \in X$ for every $e \in[X]^{<\omega}$. Note that by Kueker's Theorem mentioned above, weakly stationary is the same as stationary when $\mu=\omega$. However, it is not so for $\mu>\omega$ : see, for example, the discussion [11, end of Section 4.1]. This generalization of stationary set is due probably to Foreman, Magidor and Shelah [9], and used prominently by Woodin.

The Pressing Down Lemma for this kind of stationary sets is folklore, but we include a reference for completeness.

Lemma 2.3 (Folklore). Given a set $X$ and a regular cardinal $\mu$, for every weakly stationary set $S \subseteq[X]^{\mu}$ and any regressive function $f: S \rightarrow X$, there is a weakly stationary set $S^{\prime} \subseteq S$ such that $f \uparrow_{S^{\prime}}$ is constant.

Proof. See for example [8, p. 912, Lemma 3.3].

All along these notes we only use the notion of weakly stationary. Since for $\mu=\omega$ weakly stationary and stationary coincide, we make an abuse of language and call both just stationary, even when $\mu \geq \omega_{1}$.

\section{Semi-Stationary Reflection Principle and the Strong Tree} Property for $\omega_{2}$. We give the definitions regarding the Strong Tree Property.

Definition 3.1. Let $\lambda>\omega_{1}$ be a regular cardinal and let $\kappa \geq \lambda$. A $(\kappa, \lambda)$-tree is a system $\left\{\mathscr{F}_{a} \in P\left(2^{a}\right): a \in[\kappa]^{<\lambda}\right\}$ such that

(1) for every $a, 1 \leq\left|\mathscr{F}_{a}\right|<\lambda$,

(2) for $a, b \in[\kappa]^{<\lambda}, a \subseteq b \rightarrow \forall f \in \mathscr{F}_{b} \exists g \in \mathscr{F}_{a}\left(f \uparrow_{a}=g\right)$.

Given a $(\kappa, \lambda)$-tree $\mathscr{F}$, we order its elements in the following way: for $f, g \in \mathscr{F}, f \leq_{\mathscr{F}} g$ if and only if $g \uparrow_{\operatorname{dom}(f)}=f$. Observe that if $f \leq_{\mathscr{F}} g$, then in particular $\operatorname{dom}(f) \subseteq \operatorname{dom}(g)$. Note that $\leq \mathscr{F}$ is transitive, but it is not necessarily a tree order. We say that $f, g \in \mathscr{F}$ are compatible if there is $h \in \mathscr{F}$ such that $h \geq \mathscr{F} f, g$ (note that in a tree order, compatible is equivalent to comparable). For $A, B \subseteq \mathscr{F}$ we write $A \perp B$ if for every $f \in A$ and every $g \in B, f$ and $g$ are incompatible. Similarly, for $f, g \in \mathscr{F}$ and $A \subseteq \mathscr{F}$, we write $f \perp g$ and $f \perp A$ whenever $\{f\} \perp\{g\}$ and $\{f\} \perp A$ respectively. A cofinal branch through $\mathscr{F}$ is a function $B: \kappa \rightarrow 2$ such that $B \uparrow_{a} \in \mathscr{F}$ for every $a \in[\kappa]^{<\lambda}$.

Definition 3.2. We say that $\lambda$ has the Strong Tree Property if every $(\kappa, \lambda)$-tree has a cofinal branch for every $\kappa \geq \lambda$.

In this section, we prove that $\mathrm{CC}^{*}$ together with the negation of $\mathrm{CH}$ implies $\omega_{2}$ has the Strong Tree Property. The proofs are based on the techniques of [26] and [28]: compare, for example, our Proposition 3.1, Lemma 3.1] and 
Lemma 3.2 with Proposition 2.3, Lemma 2.4 and Lemma 2.5 in [26] and Proposition 3.1, Lemma 3.1 and Lemma 3.2 in 28 respectively.

Let $\kappa \geq \omega_{2}$ and fix a $\left(\kappa, \omega_{2}\right)$-tree $\mathscr{F}$. Fix a level enumeration surjective function $e:[\kappa]^{\omega_{1}} \times \omega_{1} \rightarrow \mathscr{F}$ such that $e(d, \xi) \in \mathscr{F}_{d}$.

We have the following:

Proposition 3.1. Given a $\left(\kappa, \omega_{2}\right)$-tree $\mathscr{F}$, let $\left\langle A_{d}: d \in[\kappa]^{\omega_{1}}\right\rangle$ be a sequence of collections of nodes such that $A_{d} \in\left[\mathscr{F}_{d}\right]^{\omega}$ for every $d \in[\kappa]^{\omega_{1}}$. Then there are $b \in[\kappa]^{\omega_{1}}$ and $E$ stationary in $[\kappa]^{\omega_{1}}$ such that for every $g \in \mathscr{F}_{b}$ and every $d \in E$, if $g$ has an extension in $\mathscr{F}_{d}$, this extension is unique.

Proof. Let $\theta$ be large enough such that $\{\mathscr{F}, e, \kappa, \ldots\}$ and all relevant parameters belong to $H_{\theta}$.

We remark the following:

REMARK 3.1. There are stationary many $M \prec H_{\theta}$ with $|M|=\aleph_{1}$ such that for every $A \in[M]^{\omega}$, there is $B \in M \cap[M]^{\omega}$ such that $B \supseteq A$.

Proof. For any $g: H_{\theta}^{<\omega} \rightarrow H_{\theta}$, build a $\subseteq$-continuous chain $\left\langle M_{\xi}: \xi<\omega_{1}\right\rangle$ of countable elementary submodels of $H_{\theta}$ such that for any $\xi \in \omega_{1}, M_{\xi}$ is closed under $g$ and $M_{\xi} \in M_{\xi+1}$. Let $M=\bigcup_{\xi<\omega_{1}} M_{\xi}$. It is easy to check that $M$ is closed under $g$ and $|M|=\omega_{1}$. Then if $A \in[M]^{\omega}$, there is $\xi \in \omega_{1}$ such that $A \subseteq M_{\xi}$, and so $M_{\xi} \in M_{\xi+1} \subseteq M$.

Let $S \subseteq\left[H_{\theta}\right]^{\omega_{1}}$ be the stationary set of $M$ 's of Remark 3.1. For any $M \in S$, let $d_{M}=M \cap \kappa$. For $g, h \in \mathscr{F}_{d_{M}}$ with $g \neq h$, choose $\alpha_{g, h} \in d_{M}$ such that $g\left(\alpha_{g, h}\right) \neq h\left(\alpha_{g, h}\right)$. Since $A_{d_{M}}$ is countable, we can apply Remark 3.1 to the set $\left\{\alpha_{g, h}: g, h \in A_{d_{M}}\right\} \in[M]^{\omega}$ to find $B_{M} \supseteq\left\{\alpha_{g, h}: g, h \in A_{d_{M}}\right\}$ with $B_{M} \in M \cap[M]^{\omega}$. Using the Pressing Down Lemma, find $B \in H_{\theta}$ and $S^{\prime} \subseteq S$ stationary such that $B_{M}=B$ for all $M \in S^{\prime}$. By Menas' Lemma, the set $E=\left\{d_{M}: M \in S^{\prime}\right\}$ is stationary in $[\kappa]^{\omega_{1}}$. Let $b=B \cap \kappa$. Then for every $f \in \mathscr{F}_{b}$, if $f$ has an extension in $\mathscr{F}_{d}$ for $d \in E$, this extension is unique.

Proposition 3.2. Let $\mathscr{F}$ be a $\left(\kappa, \omega_{2}\right)$-tree with no cofinal branches. Let $\left\langle A_{d}: d \in[\kappa]^{\omega_{1}}\right\rangle$ be a sequence of collections of nodes such that $A_{d} \in\left[\mathscr{F}_{d}\right]^{\omega}$ for every $d \in[\kappa]^{\omega_{1}}$. Let $\theta$ be large enough such that $\{\mathscr{F}, e, \kappa, \ldots\}$ and all relevant parameters belong to $H_{\theta}$, and let $N \prec H_{\theta}$ be such that $|N|=\aleph_{1}$ and $N \supseteq \omega_{1}$. Then for every $f \in \mathscr{F}$ with $\operatorname{dom}(f) \supseteq N \cap \kappa$, there is $d \in N \cap[\kappa]^{\omega_{1}}$ such that $f \uparrow_{d} \notin A_{d}$.

Proof. Suppose otherwise. Fix $f \in \mathscr{F}$ with $\operatorname{dom}(f) \supseteq N \cap \kappa$ such that $f \uparrow_{d} \in A_{d}$ for every $d \in N \cap[\kappa]^{\omega_{1}}$. Apply Proposition 3.1 to find $b \in[\kappa]^{\omega_{1}}$ and a stationary set $E \subseteq[\kappa]^{\omega_{1}}$ such that one can define for every $g \in \mathscr{F}_{b}$ a function $F_{g}: E \rightarrow \mathscr{F}$, where $F_{g}(d)$ is the unique extension in $\mathscr{F}_{d}$ of $g$ if the extension exists, or the empty set otherwise. Observe that by elementarity, we can take $E, b \in N$ such that $F_{g}$ is defined in $N$ for every $g \in \mathscr{F}_{b} \cap N$. 
Furthermore, since $N$ is closed under the level enumeration function, and $\omega_{1} \cup\{b\} \subseteq N$, we get $\mathscr{F}_{b} \subseteq N$. In particular $f \uparrow_{b} \in N$, and therefore $F_{f \uparrow_{b}}$ is defined in $N$. To simplify notation, let $F=F_{\left.f\right|_{b}}$, and let $B=\bigcup_{d \in E} F(d)$ (which is also defined in $N$ ).

Observe that for any $d \in E$, we get $F(d) \neq \emptyset$, since $F(d)=f \uparrow_{d}$. Also for $d, d^{\prime} \in E, F(d)$ and $F\left(d^{\prime}\right)$ are $\leq \mathscr{F}$-comparable since $F(d)=f \uparrow_{d}$ and $F\left(d^{\prime}\right)=f \uparrow_{d^{\prime}}$, By our initial supposition of the proof, for every $d \in[\kappa]^{\omega_{1}} \cap N$, $B \uparrow_{d}\left(=f \uparrow_{d}\right) \in \mathscr{F}$. Therefore, by elementarity, $B$ defines in $N$ a cofinal branch in $\mathscr{F}$, a contradiction.

Lemma 3.1. $\left(\mathrm{CC}^{*}\right)$ Let $\mathscr{F}$ be a $\left(\kappa, \omega_{2}\right)$-tree with no cofinal branches. Then there are arbitrarily large $\theta$ such that for every countable $M \prec H_{\theta}$ there are $M_{0}, M_{1} \prec H_{\theta}$ countable and $a_{0} \in M_{0} \cap[\kappa]^{\omega_{1}}, a_{1} \in M_{1} \cap[\kappa]^{\omega_{1}}$ with

(1) $M \cap \omega_{1}=M_{0} \cap \omega_{1}=M_{1} \cap \omega_{1}$,

(2) $\mathscr{F}_{a_{0}} \cap M_{0} \perp \mathscr{F}_{a_{1}} \cap M_{1}$.

Proof. Apply $\mathrm{CC}^{*}$ to find $\theta$ sufficiently large such that all relevant parameters belong to $H_{\theta}$ and such that $\mathrm{CC}(\kappa, \theta)$ holds. Take $M \prec H_{\theta}$ countable. Our goal is to find $a_{0}, a_{1}, M_{0}, M_{1}$ such that (1) and (2) of the present lemma hold.

Let $\theta^{\prime}>\theta$ be sufficiently large such that $M, \mathscr{F}, e, H_{\theta}$ and all relevant parameters are members of $H_{\theta^{\prime}}$ and such that $\mathrm{CC}(\kappa, \theta)$ holds in $H_{\theta^{\prime}}$. Take $N \prec H_{\theta^{\prime}}$ of size $\aleph_{1}$ with $\omega_{1} \subseteq N$ and containing all relevant parameters such as $M$ and $\mathscr{F}$. Let $a=N \cap \kappa$. To build $a_{1}$ and $M_{1}$, simply apply CC* (outside $N$ ) to find $M_{1} \prec H_{\theta}$ and $a_{1} \in M_{1} \cap[\kappa]^{\omega_{1}}$ such that $a_{1} \supseteq a$ and $M_{1} \sqsupseteq M$. We will show later that $M_{1}, a_{1}$ are the ones that we are looking for. To find $a_{0}$ and $M_{0}$ we need a little more. First we prove the following:

Claim 3.1. Let $K$ be a countable elementary submodel of $H_{\theta}$ with $K \in N$ and let $b \in[\kappa]^{\omega_{1}}$ with $b \supseteq a$. Then for every $f \in \mathscr{F}_{b}$, there is $K^{*} \sqsupseteq K$ with $K^{*} \in N$ and $c \in K^{*} \cap[\kappa]^{\omega_{1}}$ such that $f \perp K^{*} \cap \mathscr{F}_{c}$.

Proof. Assume otherwise. Take $f \in \mathscr{F}_{b}$ such that for any $K^{*} \in N$ with $K^{*} \sqsupseteq K$ and for all $c \in K^{*} \cap[\kappa]^{\omega_{1}}$, there is $g_{c} \in K^{*} \cap \mathscr{F}_{c}$ compatible with $f$.

REMARK 3.2. For every $c \in K^{*} \cap[\kappa]^{\omega_{1}}, f$ and $g_{c}$ are not only compatible, but indeed $f \geq g_{c}$.

Proof. This follows directly by showing that $c \subseteq a(\subseteq b)$ for every $c \in$ $K^{*} \cap[\kappa]^{\omega_{1}}$. Since $K^{*} \in N$ and $K^{*}$ is countable, we have $K^{*} \subseteq N$. So if $c \in K^{*} \cap[\kappa]^{\omega_{1}}$, in particular $c \in N$. Since $\omega_{1} \subseteq N$, we also have $c \subseteq N$, and therefore $c \subseteq N \cap \kappa=a$.

Working in $N$ and using the fact that $\mathrm{CC}^{*}$ holds in $N$, build a sequence $\left\langle\left(K_{d}, d^{\prime}\right): d \in[\kappa]^{\omega_{1}}\right\rangle$ such that $K_{d}$ is a countable submodel of $H_{\theta}, K_{d} \sqsupseteq K$, 
$d^{\prime} \supseteq d$ and $d^{\prime} \in K_{d} \cap[\kappa]^{\omega_{1}}$ for every $d \in[\kappa]^{\omega_{1}}$. For $d \in[\kappa]^{\omega_{1}}$, define

$$
A_{d}=\left\{h \in \mathscr{F}_{d}: \exists g \in K_{d} \cap \mathscr{F}_{d^{\prime}}(g \geq \mathscr{F} h)\right\} .
$$

Observe that whenever $d \subseteq d^{\prime}$, if $h_{0}, h_{1} \in \mathscr{F}_{d}$ and $g \in \mathscr{F}_{d^{\prime}}$ with $h_{0}, h_{1} \leq \mathscr{F} g$, then $h_{0}=h_{1}$ (since $\left.h_{0}=g \uparrow_{\operatorname{dom}\left(h_{0}\right)}=g \uparrow_{d}=g \uparrow_{\operatorname{dom}\left(h_{1}\right)}=h_{1}\right)$. Therefore the cardinality of $A_{d}$ is at most the cardinality of $K_{d}$, which is countable. We can now apply Proposition 3.2 to $N, f$ and $\left\langle A_{d}: d \in[\kappa]^{\omega_{1}}\right\rangle$ to find $d \in N \cap[\kappa]^{\omega_{1}}$ such that

$$
f \uparrow_{d} \notin A_{d} .
$$

By our assumption at the beginning of the proof of this claim, and by Remark 3.2, there is $g \in K_{d} \cap \mathscr{F}_{d^{\prime}}$ with $g \leq_{\mathscr{F}} f$. By definition of $A_{d}$, we have $g \uparrow_{d} \in A_{d}$. But $g{\Upsilon_{d}}_{d}=\left(f \uparrow_{d^{\prime}}\right) \Upsilon_{d}=f \uparrow_{d}$, contradicting (1).

We now continue with the proof of Lemma 3.1. Let $\left\{f_{n}: n \in \omega\right\}$ be an enumeration of $M_{1} \cap \mathscr{F}_{a_{1}}$. Then, applying Claim 3.1, build a $\sqsubseteq$-increasing sequence $\langle M(n): n \in \omega\rangle$ and a sequence $\langle c(n): n \in \omega\rangle$ such that for every $n \in \omega$, we have $M(n) \sqsupseteq M, c(n) \in M(n) \cap[\kappa]^{\omega_{1}}$ and

$$
f_{n} \perp M(n) \cap \mathscr{F}_{c(n)} \text {. }
$$

Using $\mathrm{CC}^{*}$, find $M_{0} \sqsupseteq \bigcup_{n \in \omega} M(n)$ with $M_{0} \prec H_{\theta}$ and $a_{0} \in M_{0} \cap[\kappa]^{\omega_{1}}$ such that $a_{0} \supseteq \bigcup_{n \in \omega} c_{n}$. We claim that (2) of Lemma 3.1 holds for $a_{0}, a_{1}, M_{0}$ and $M_{1}$, i.e. $\mathscr{F}_{a_{0}} \cap M_{0} \perp \mathscr{F}_{a_{1}} \cap M_{1}$. To see that, take $n \in \omega$ and $g \in M_{0} \cap \mathscr{F}_{a_{0}}$; we will show that $f_{n} \perp g$. Observe that

$$
M(n) \cap \mathscr{F}_{c(n)}=M_{0} \cap \mathscr{F}_{c(n)},
$$

since $c(n) \in M(n) \subseteq M_{0}, M_{0} \cap \omega_{1}=M(n) \cap \omega_{1}$ and the enumeration function $e$ is in both $M_{0}$ and $M(n)$. Since $g \in M_{0} \cap \mathscr{F}_{a_{0}}$ and $c(n) \in M(n) \subseteq M_{0}$, we have $g \uparrow_{c(n)} \in M_{0} \cap \mathscr{F}_{c(n)}$. Therefore, by (3), we get $g \uparrow_{c(n)} \in M(n) \cap \mathscr{F}_{c(n)}$. Using (2), we obtain $f_{n} \perp g \uparrow_{c(n)}$, and therefore $f_{n} \perp g$.

We have the following:

Lemma 3.2. $\left(\mathrm{CC}^{*}\right)$ Let $\mathscr{F}$ be a $\left(\kappa, \omega_{2}\right)$-tree with no cofinal branches. For $\lambda$ sufficiently large, if the set

$$
S_{\mathscr{F}}=\left\{M \in\left[H_{\lambda}\right]^{\omega}: \exists b \in[\kappa]^{\omega_{1}} \forall f \in \mathscr{F}_{b} \exists a \in M \cap[b]^{\omega_{1}}\left(f \uparrow_{a} \notin M\right)\right\}
$$

is nonstationary, then $\mathrm{CH}$ holds.

Proof. Suppose $S_{\mathscr{F}}$ is nonstationary, and let $F:\left[H_{\lambda}\right]^{<\omega} \rightarrow H_{\lambda}$ be a function such that if $M \in\left[H_{\lambda}\right]^{\omega}$ is closed under $F$, then $M \notin S_{\mathscr{F}}$. As before, let $e:[\kappa]^{\omega_{1}} \times \omega_{1} \rightarrow \mathscr{F}$ be a surjective function such that $e(a, \xi) \in \mathscr{F}_{a}$ for every $\xi \in \omega_{1}$. Let $\theta$ be sufficiently large such that $\mathscr{F}, S_{\mathscr{F}}, F, e$ and all relevant parameters are in $H_{\theta}$ and the conclusion of Lemma 3.1 holds.

Using Lemma 3.1 , build a binary tree $\left\langle M_{\sigma}\right\rangle_{\sigma \in 2}<\omega$ of countable elementary submodels of $H_{\theta}$ with the property that for every $\sigma \in 2^{<\omega}$, 
(1) $M_{\sigma} \cap \omega_{1}=M_{\sigma \frown 0} \cap \omega_{1}=M_{\sigma \frown 1} \cap \omega_{1}$, and

(2) there exist $a_{0} \in M_{\sigma \frown 0} \cap[\kappa]^{\omega_{1}}$ and $a_{1} \in M_{\sigma \frown 1} \cap[\kappa]^{\omega_{1}}$ such that $\mathscr{F}_{a_{0}} \cap M_{\sigma \frown 0} \perp \mathscr{F}_{a_{1}} \cap M_{\sigma \frown 1}$.

For every $r \in 2^{\omega}$, let $M_{r}=\bigcup_{n \in \omega} M_{r \mid n}$. Let $b \in[\kappa]^{\omega_{1}}$ be such that $b \supseteq a$ for every $a \in M_{\sigma} \cap[\kappa]^{\omega_{1}}$ and every $\sigma \in 2^{<\omega}$. Since $M_{r} \prec H_{\theta}$ and $F \in M_{r}$, $M_{r}$ is closed under $F$, we have $M_{r} \cap \kappa \notin S_{\mathscr{F}}$. So we can choose $f_{r} \in \mathscr{F}_{b}$ such that $f_{r} \uparrow_{a} \in M_{r}$ for every $a \in M_{r} \cap[b]^{\omega_{1}}$.

Claim 3.2. The map $r \mapsto f_{r}$ is an injection from $2^{\omega}$ to $\mathscr{F}_{b}$ (and therefore CH holds).

Proof. Let $r_{0}, r_{1} \in 2^{\omega}$ with $r_{0} \neq r_{1}$ and denote by $f_{i}$ the node $f_{r_{i}}$ for $i \in$ $\{0,1\}$. We will find two predecessors of $f_{0}$ and $f_{1}$ that are incompatible. Let $n \in \omega$ be such that $r_{0} \Upsilon_{n}=r_{1} \Upsilon_{n}=\sigma$, and $r_{0} \Upsilon_{n+1} \neq r_{1} \Upsilon_{n+1}$. Without loss of generality, suppose $r_{i}(n)=i$ for $i \in\{0,1\}$. By the construction of our binary tree, we can take $a_{0} \in M_{r_{0}\left\lceil_{n+1}\right.}$ and $a_{1} \in M_{r_{1}\left\lceil_{n+1}\right.}$ such that $\mathscr{F}_{a_{0}} \cap M_{r_{0}\left\lceil_{n+1}\right.} \perp$ $\mathscr{F}_{a_{1}} \cap M_{r_{1}\left\lceil_{n+1}\right.}$. However, observe that for $i \in\{0,1\}, a_{i} \in M_{r_{i} \uparrow_{n+1}} \subseteq M_{r_{i}}$, and so $f_{i} \uparrow_{a_{i}} \in M_{r_{i} \uparrow_{n+1}}$. Therefore, $f_{0} \uparrow_{a_{0}}$ and $f_{1} \uparrow_{a_{1}}$ are incompatible, and so are $f_{0}$ and $f_{1}$.

This finishes the proof of Lemma 3.2 .

We are ready to prove the main theorem of this section.

Theorem 3.1. $\left(\mathrm{CC}^{*}\right)$ If $\mathrm{CH}$ does not hold, then $\omega_{2}$ has the Strong Tree Property.

Proof. Assume $\mathrm{CH}$ does not hold, but there is a $\left(\kappa, \omega_{2}\right)$-tree $\mathscr{F}$ with no cofinal branches. From Lemma 3.2, for $\lambda$ sufficiently large, the set $S_{\mathscr{F}}$ is stationary in $\left[H_{\lambda}\right]^{\omega}$, and in particular it is semi-stationary. Without loss of generality, we can assume that every set in $S_{\mathscr{F}}$ is closed under $e$. Since $\mathrm{CC}^{*}$ and SSR are equivalent [5, Theorem 5.7], we can apply SSR to obtain $X \in\left[H_{\lambda}\right]$ with $X \supseteq \omega_{1}$ such that $[X]^{\omega} \cap S_{\mathscr{F}}$ is semi-stationary. Let

$$
S=\left\{x \in[X]^{\omega}: \exists M_{x} \in S_{\mathscr{F}} \cap[X]^{\omega}\left(x \sqsupseteq M_{x}\right)\right\},
$$

which is stationary by definition of semi-stationary set. Take a stationary set $S^{\prime} \subseteq S$ of size $\omega_{1}\left(^{1}\right)$. For $x \in S^{\prime}$, using the definition of $S_{\mathscr{F}}$, choose $b_{x} \in[\kappa]^{\omega_{1}}$ such that for every $f \in \mathscr{F}_{b x}$, there is $a \in M_{x} \cap\left[b_{x}\right]^{\omega_{1}}$ with $f \uparrow_{a} \notin M_{x}$. Let $b=\bigcup_{x \in S^{\prime}} b_{x}$ (and so $|b|=\omega_{1}$ ). Fix $f \in \mathscr{F}_{b}$. Then for $x \in S^{\prime}$, we can choose $a_{x} \in M_{x} \cap\left[b_{x}\right]^{\omega_{1}}$ such that

$$
\left(f \uparrow_{b_{x}}\right) \uparrow_{a_{x}}=f \uparrow_{a_{x}} \notin M_{x}
$$

Apply the Pressing Down Lemma to find $a \in[\kappa]^{\omega_{1}}$ and a stationary set $S^{\prime \prime} \subseteq S^{\prime}$ such that $a_{x}=a$ for every $x \in S^{\prime \prime}$. Observe that since $S^{\prime \prime}$ is

$\left({ }^{1}\right)$ For example, let $h: X \rightarrow \omega_{1}$ be a bijection. So the set $\left\{h^{-1}[\alpha]: \alpha \in \omega_{1} \backslash \omega\right\}$ is a club of size $\omega_{1}$, and take its intersection with $S$. 
stationary in $[X]^{\omega}$, it is in particular cofinal in $[X]^{\omega}$, and since $X \supseteq \omega_{1}$, we have $\bigcup_{x \in S^{\prime \prime}}\left(x \cap \omega_{1}\right)=\omega_{1}$. Therefore we can fix $x \in S^{\prime \prime}$ and $\xi \in x$ such that $e(a, \xi)=f \uparrow_{a}$. However, $M_{x}$ is closed under $e$, and $M_{x} \cap \omega_{1}=x \cap \omega_{1}$ (since $\left.x \sqsupseteq M_{x}\right)$, and so $e(\xi, a) \in M_{x}$, contradicting (4).

4. Square sequences. Given a set $A$ of ordinals, we denote by $\operatorname{Lim}(A)$ the collection of limit points of $A$, i.e. $\alpha \in \operatorname{Lim}(A)$ if $\alpha>0$ and $\sup (A \cap \alpha)=$ $\alpha$ (so in particular, $\alpha$ is a limit ordinal). Observe also that if $A \subseteq B$, we have $\operatorname{Lim}(A) \subseteq \operatorname{Lim}(B)$.

We recall a two-cardinal version $\square(\lambda, \mu)$ of the square principle.

Definition 4.1. Given a regular cardinal $\lambda$ and a cardinal $\mu \leq \lambda,\left\langle\mathscr{C}_{\alpha}\right.$ : $\alpha \in \operatorname{Lim}(\lambda)\rangle$ is a $(\lambda, \mu)$-square sequence or a $\square(\lambda, \mu)$-sequence if

(1) $1 \leq\left|\mathscr{C}_{\alpha}\right| \leq \mu$,

(2) for every $C \in \mathscr{C}_{\alpha}, C$ is a closed and unbounded subset of $\alpha$,

(3) for every $C \in \mathscr{C}_{\beta}$, if $\alpha \in \operatorname{Lim}(C)$, then $C \cap \alpha \in \mathscr{C}_{\beta}$.

Given a set $C \subseteq \lambda$, we say that $C$ trivializes a $(\lambda, \mu)$-square sequence $\left\langle\mathscr{C}_{\alpha}\right.$ : $\alpha \in \operatorname{Lim}(\lambda)\rangle$ if $C \cap \alpha \in \mathscr{C}_{\alpha}$ for every $\alpha \in \operatorname{Lim}(C)$.

We say that the principle $\square(\lambda, \mu)$ holds if there is a $(\lambda, \mu)$-square sequence $\left\langle\mathscr{C}_{\alpha}: \alpha \in \operatorname{Lim}(\lambda)\right\rangle$ that is trivialized by no club.

We first give some lemmas which describe some properties of square sequences of the form $\square(\lambda, \mu)$.

Lemma 4.1. For a $(\lambda, \mu)$-square sequence $\left\langle\mathscr{C}_{\alpha}: \alpha \in \operatorname{Lim}(\lambda)\right\rangle$ the following are equivalent:

(1) There is a club $D \subseteq \lambda$ trivializing the sequence.

(2) There is $C \subseteq \lambda$ such that $\operatorname{Lim}(C)$ is unbounded in $\lambda$ and a sequence $\left\langle C_{\gamma}: \gamma \in \operatorname{Lim}(C)\right\rangle$ such that for every $\gamma \in \operatorname{Lim}(C), C_{\gamma} \in \mathscr{C}_{\gamma}$, and for $\alpha, \beta \in \operatorname{Lim}(C)$, if $\alpha<\beta$ then $C_{\alpha}=C_{\beta} \cap \alpha$.

Proof. (1) $\Rightarrow(2)$. Just set $C=D$ and $C_{\gamma}=D \cap \gamma$ for every $\gamma \in \operatorname{Lim}(D)$.

$(2) \Rightarrow(1)$. Take $C$ as in the assumption, and set $D=\bigcup_{\alpha \in \operatorname{Lim}(C)} C_{\alpha}$. We will show that $D \cap \alpha \in \mathscr{C}_{\alpha}$ for every $\alpha \in \operatorname{Lim}(D)$.

Take $\alpha \in \operatorname{Lim}(D)$ and $\beta \in \operatorname{Lim}(C)$ such that $\alpha \in C_{\beta}$. Using the properties of the sequence $\left\langle C_{\gamma}: \gamma \in \operatorname{Lim}(C)\right\rangle$, it is not difficult to show that $\alpha$ is also a limit point of $C_{\beta}$, and so $C_{\beta} \cap \alpha \in \mathscr{C}_{\beta}$. Therefore, it is enough to show that $D \cap \alpha=C_{\beta} \cap \alpha$. Note that already $C_{\beta} \subseteq D$. So $C_{\beta} \cap \alpha \subseteq D \cap \alpha$. Therefore, it remains to show that $D \cap \alpha \subseteq C_{\beta} \cap \alpha$. Observe that by the properties of $\left\langle C_{\gamma}: \gamma \in \operatorname{Lim}(C)\right\rangle$, we can easily verify $C_{\gamma} \cap \alpha \subseteq C_{\beta} \cap \alpha$ for every $\gamma \in \operatorname{Lim}(C)$, and therefore $D \cap \alpha=C_{\beta} \cap \alpha$.

We show that if $\operatorname{Lim}(C)$ is unbounded, then $D$ is a club: To show that $D$ is unbounded, take $\beta<\lambda$. Since $\operatorname{Lim}(C)$ is unbounded in $\lambda$, there is $\alpha>\beta$ 
with $\alpha \in \operatorname{Lim}(C)(\subseteq \operatorname{Lim}(\lambda))$ and $C_{\alpha}$ unbounded in $\alpha$. To show that $D$ is closed, take an increasing sequence $\left\langle\beta_{\xi}: \xi<\gamma\right\rangle$ of elements of $D$ with $\gamma<\lambda$. Let $\beta=\sup \left\{\beta_{\xi}: \xi<\gamma\right\}$. We wish to show that $\beta \in D$. For every $\xi<\gamma$, there is $\alpha_{\xi} \in \operatorname{Lim}(C)$ such that $\beta_{\xi} \in C_{\alpha_{\xi}}$. Let $\alpha=\sup \left\{\alpha_{\xi}: \xi<\gamma\right\}<\lambda$. Since $\operatorname{Lim}(C)$ is unbounded in $\lambda$, let $\eta \in \operatorname{Lim}(C)$ with $\eta>\alpha$. By the properties of $C$, we have $C_{\eta} \cap \alpha_{\xi}=C_{\alpha_{\xi}}$ for every $\xi<\gamma$, and so $\left\{\beta_{\xi}: \xi<\gamma\right\} \subseteq C_{\eta}$. Since $C_{\eta}$ is closed, $\sup \left\{\beta_{\xi}: \xi \in \gamma\right\} \in C_{\eta} \subseteq D$.

REMARK 4.1. Let $\lambda$ be a regular uncountable cardinal, and let $\left\langle\mathscr{C}_{\beta}\right.$ : $\beta \in \operatorname{Lim}(\lambda)\rangle$ be a $\square(\lambda, \mu)$-sequence with $\lambda>\operatorname{cof}(\mu)^{+}$. For $\beta<\mu$, let $\mathscr{C}_{\beta}=\left\{C_{\beta}^{\xi}: \xi<\mu\right\}$. Then for every $\beta \in \lambda \cap \operatorname{Cof}(>\mu)$, there is $\alpha_{\beta}<\beta$ such that for every $C_{\xi}, C_{\eta} \in \mathscr{C}_{\beta}$, if $C_{\xi} \neq C_{\eta}$, then $C_{\xi} \cap \alpha_{\beta} \neq C_{\eta} \cap \alpha_{\beta}$.

Proof. For $C_{\xi}, C_{\eta} \in \mathscr{C}_{\beta}$ with $C_{\xi} \neq C_{\eta}$, choose $\alpha_{\{\xi, \eta\}}<\beta$ such that $C_{\xi} \cap \alpha_{\{\xi, \eta\}} \neq C_{\eta} \cap \alpha_{\{\xi, \eta\}}$. If $C_{\xi}=C_{\eta}$, let $\alpha_{\{\xi, \eta\}}$ be just any $\alpha$ below $\beta$. Let $\alpha_{\beta}=\sup \left\{\alpha_{\{\xi, \eta\}}:\{\xi, \eta\} \in[\mu]^{2}\right\}$. Since $\operatorname{cof}(\beta)>\mu$, we have $\alpha_{\beta}<\beta$, and therefore $C_{\xi} \cap \alpha_{\beta} \neq C_{\eta} \cap \alpha_{\beta}$ for every $\{\xi, \eta\} \in[\mu]^{2}$ with $C_{\xi} \neq C_{\eta}$.

LEMMA 4.2. Let $\lambda$ be a regular uncountable cardinal, and let $\left\langle\mathscr{C}_{\beta}\right.$ : $\beta \in \operatorname{Lim}(\lambda)\rangle$ be $a \square(\lambda, \mu)$-sequence with $\lambda>\operatorname{cof}(\mu)^{+}$such that no club trivializes this sequence. For $\beta<\mu$, let $\mathscr{C}_{\beta}=\left\{C_{\beta}^{\xi}: \xi<\mu\right\}$. For any set $X \subseteq \lambda$ such that $X \cap \operatorname{Cof}(>\mu)$ is stationary, and for every $M \prec H_{\theta}$ with $\theta$ sufficiently large and $\left\{X,\left\langle\mathscr{C}_{\beta}: \beta \in \operatorname{Lim}(\lambda)\right\rangle\right\} \cup \mu \subseteq M$, if $\delta=\sup (M \cap \lambda)$, then for every $\xi \in \mu$, the set

$$
\left\{\alpha \in X \cap M: \alpha \notin \operatorname{Lim}\left(C_{\delta}^{\xi}\right)\right\}
$$

is unbounded in $\delta$.

Proof. Suppose it is not the case. Then there are $\xi^{*} \in \mu$ and $\gamma \in M \cap \lambda$ such that $X \cap M \backslash \gamma \subseteq \operatorname{Lim}\left(C_{\delta}^{\xi^{*}}\right)$. Let $X_{0}=X \backslash \gamma$, so in particular $X_{0} \in M$, and similarly $\operatorname{Lim}\left(X_{0}\right) \in M$. Observe also that $X_{0} \cap \operatorname{Cof}(>\mu)$ is stationary. Applying Fodor's Lemma and Remark 4.1, there is $\alpha \in \lambda$ and a stationary subset $X_{1} \subseteq X_{0} \cap \operatorname{Cof}(>\mu)$ such that $\alpha_{\beta}=\alpha$ for every $\beta \in X_{1}$. Since $X_{0} \in M$, by elementarity we can take $\alpha, X_{1} \in M$.

Remark 4.2. $C_{\delta}^{\xi^{*}} \cap \alpha \in M$.

Proof. Pick any $\beta \in \operatorname{Lim}\left(X_{1}\right) \cap M \backslash \alpha$. As $\operatorname{Lim}\left(X_{1}\right) \cap M \subseteq X_{0} \cap M \subseteq$ $\operatorname{Lim}\left(C_{\delta}^{\xi^{*}}\right)$, there is $\xi_{\beta} \in \mu(\subseteq M)$ such that $C_{\delta}^{\xi^{*}} \cap \beta=C_{\beta}^{\xi_{\beta}}$. But then $C_{\delta}^{\xi^{*}} \cap \alpha=$ $C_{\delta}^{\xi^{*}} \cap(\beta \cap \alpha)=\left(C_{\delta}^{\xi^{*}} \cap \beta\right) \cap \alpha=C_{\beta}^{\xi_{\beta}} \cap \alpha$. Since $\alpha, \beta, \xi_{\beta} \in M$, the set $C_{\beta}^{\xi_{\beta}}$ is defined in $M$, and so $C_{\delta}^{\xi^{*}} \cap \alpha \in M$.

To simplify notation, write $C^{*}=C_{\delta}^{\xi^{*}} \cap \alpha$, so by Remark 4.2 , $C^{*} \in M$.

Claim 4.1. For every $\beta \in \operatorname{Lim}\left(X_{1}\right)$, there is a unique $\xi_{\beta}$ such that $C_{\beta}^{\xi_{\beta}} \cap \alpha=C^{*}$. 
Proof. By the elementarity of $M$, it suffices to prove that Claim 4.1 holds in $M$. To show existence, using $\operatorname{Lim}\left(X_{1}\right) \cap M \subseteq \operatorname{Lim}\left(C_{\delta}^{\xi^{*}}\right)$, pick $\xi_{\beta}$ such that $C_{\delta}^{\xi^{*}} \cap \beta=C_{\beta}^{\xi_{\beta}}$. Then $C_{\beta}^{\xi_{\beta}} \cap \alpha=\left(C_{\delta}^{\xi^{*}} \cap \beta\right) \cap \alpha=C_{\delta}^{\xi^{*}} \cap(\beta \cap \alpha)=C_{\delta}^{\xi^{*}} \cap \alpha=C^{*}$. To show uniqueness, take $\xi_{\beta}, \eta_{\beta} \in \mu$ such that $C_{\beta}^{\xi_{\beta}} \neq C_{\beta}^{\eta_{\beta}}$. Since $\beta \in X_{1}$, we have $C_{\beta}^{\xi_{\beta}} \cap \alpha \neq C_{\beta}^{\eta_{\beta}} \cap \alpha$, so both cannot be equal to $C^{*}$.

Define now $C_{\beta}=C_{\beta}^{\xi_{\beta}}$ for $\beta \in X_{1}$. Then the sequence $\left\langle C_{\beta}: \beta \in \operatorname{Lim}\left(X_{1}\right)\right\rangle$ is in $M$. Observe that for every $\gamma, \beta \in M \cap \operatorname{Lim}\left(X_{1}\right)$, if $\gamma<\beta$ we have $C_{\beta} \cap \gamma=\left(C_{\delta}^{\xi^{*}} \cap \beta\right) \cap \gamma=C_{\delta}^{\xi^{*}} \cap \gamma=C_{\gamma}$, contradicting Lemma 4.1.

In this section, we prove that assuming SSR, we can have the negation of $\square(\lambda, \omega)$ for every regular cardinal $\lambda \geq \omega_{2}$.

For a set $A$ of ordinals, define $\sup ^{+}(A)=\sup \{\alpha+1: \alpha \in A\}$. We will use the following useful implications of SSR given by Sakai-Veličković. Fix a regular cardinal $\lambda \geq \omega_{2}$. For countable sets of ordinals $x$ and $y$, we write $x \sqsubseteq^{*} y$ if

- $x \sqsubseteq y$,

- $\sup ^{+}(x)=\sup ^{+}(y)$,

- $\sup ^{+}(x \cap \gamma)=\sup ^{+}(y \cap \gamma)$ for all $\gamma \in E_{\omega_{1}}^{\lambda} \cap x$.

Given $X \subset[\lambda]^{\omega}$ for some $\lambda \geq \omega_{1}$, we say that $X$ is weakly full if $X$ is upward closed under $\sqsubseteq^{*}$.

Lemma 4.3 ([19, Lemma 2.2]). Let $\lambda \geq \omega_{2}$. Suppose there is a weakly full stationary $X \subseteq[\lambda]^{\omega}$ such that for every $I \in[\lambda]^{\omega_{1}}$ with $\omega_{1} \subseteq I$, there is

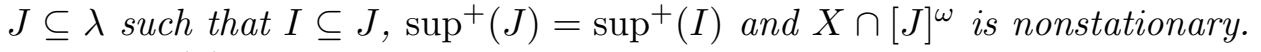
Then $\operatorname{SSR}(\lambda)$ fails.

Sakai and Veličković also present a game which will be used to construct a weakly full stationary set. Let $\lambda$ be a regular cardinal $\geq \omega_{2}$. For a function $F:[\lambda]^{<\omega} \rightarrow \lambda$ let $G_{1}(\lambda, F)$ be the following game of length $\omega$ :

\begin{tabular}{ccccccccc}
\hline I & $\alpha_{0}$ & $\gamma_{0}$ & $\alpha_{1}$ & $\gamma_{1}$ & $\cdots$ & $\alpha_{n}$ & $\gamma_{n}$ & $\cdots$ \\
II & $\beta_{0}$ & $\beta_{1}$ & $\cdots$ & $\beta_{n}$ & $\cdots$ \\
\hline
\end{tabular}

I and II in turn choose ordinals $<\lambda$. In the $n$th stage, first I chooses $\alpha_{n}$, then II chooses $\beta_{n}$, and then I again chooses $\gamma_{n}>\beta_{n}$, with $\gamma_{n}$ of cofinality $\omega_{1}$. I wins if

$$
\operatorname{cl}_{F}\left(\left\{\gamma_{n}: n \in \omega\right\}\right) \cap\left[\alpha_{m}, \gamma_{m}\right)=\emptyset
$$

for every $m \in \omega$, where $\operatorname{cl}_{F}(A)$ denotes the closure of the set $A$ under $F$. Otherwise, II wins.

Lemma 4.4 ([19, Lemma 2.3]). Let $\lambda$ be a regular cardinal $\geq \omega_{2}$ and let $F:[\lambda]^{<\omega} \rightarrow \lambda$. Then I has a winning strategy in the game $G_{1}(\lambda, F)$. 
Now we state our theorem.

TheOREM 4.1. For every regular cardinal $\lambda \geq \omega_{2}, \operatorname{SSR}(\lambda)$ implies the negation of $\square(\lambda, \omega)$.

Proof. Assuming that $\square(\lambda, \omega)$ holds, we will show that $\operatorname{SSR}(\lambda)$ fails.

Let $\left\langle\mathscr{C}_{\alpha}: \alpha \in \operatorname{Lim}(\lambda)\right\rangle$ be a $(\lambda, \omega)$-square sequence that is trivialized by no club subset of $\lambda$. Without loss of generality, we can assume $\left|\mathscr{C}_{\alpha}\right|=\omega$ for every $\alpha \in \operatorname{Lim}(\lambda)$. Let $\left\langle C_{\alpha}^{n}: n<\omega\right\rangle$ enumerate $\mathscr{C}_{\alpha}$.

Let $X$ be the set of all $x \in[\lambda]^{\omega}$ which have limit order type and there is a sequence $\left\langle\xi_{n}^{x}: n<\omega\right\rangle$ of ordinals below $\sup (x)$ such that for all $n \in \omega$,

(1) $\sup \left(x \cap C_{\sup ^{+}(x)}^{n}\right) \leq \xi_{n}^{x}$,

(2) $\operatorname{cof}(\min (x \backslash \beta))=\omega_{1}$ for all $\beta \in C_{\sup ^{+}(x)}^{n} \backslash \xi_{n}^{x}$.

It is not hard to check that $X$ is weakly full. We have the following.

Lemma 4.5. $X$ is stationary in $[\lambda]^{\omega}$.

Proof. Let $F:[\lambda]^{<\omega} \rightarrow \lambda$. We will find $x \in X$ closed under $F$. By Lemma 4.4. fix a winning strategy $\tau$ of I for $G_{1}(\lambda, F)$. Moreover let $C$ be the set of all limit ordinals $<\lambda$ closed under $\tau$ and $F$. Note that $C$ is club in $\lambda$.

Let $\theta$ be sufficiently large such that $H_{\theta}$ has all the relevant parameters. We are going to build inductively a sequence $\left\langle\mathfrak{M}_{n}: n \in \omega\right\rangle$ of structures of $H_{\theta}$ as follows: Fix a well-order $<$ of $H_{\theta}$, let $\mathfrak{M}_{0}=\left\langle H_{\theta} ; \in,<,\left\langle\mathscr{C}_{\alpha}: \alpha \in\right.\right.$ $\operatorname{Lim}(\lambda)\rangle, F, C, \ldots\rangle$, let $\left\langle M_{\xi}^{0}: \xi<\lambda\right\rangle$ be a strictly continuous $\subseteq$-increasing sequence of elementary submodels of $\mathfrak{M}_{0}$ of size $<\lambda$, and define $D_{0}=$ $\left\{\sup \left(M_{\xi}^{0} \cap \lambda\right): \xi<\lambda\right\}$. Observe that $D_{0}$ is a club in $\lambda$ and $D_{0} \in H_{\theta}$. Suppose we have defined a structure $\mathfrak{M}_{n}$ of $H_{\theta}$ and a strictly continuous $\subseteq$-increasing sequence $\left\langle M_{\xi}^{n}: \xi<\lambda\right\rangle$ of elementary submodels of $\mathfrak{M}_{n}$ of size $<\lambda$. Define $D_{n}=\left\{\sup \left(M_{\xi}^{n} \cap \lambda\right): \xi<\lambda\right\}$, so that $D_{n}$ is a club in $\lambda$ with $D_{n} \in H_{\theta}$. Let $\mathfrak{M}_{n+1}=\left\langle H_{\theta} ; \in,<,\left\langle\mathscr{C}_{\alpha}: \alpha \in \operatorname{Lim}(\lambda)\right\rangle, F, C, D_{0}, \ldots, D_{n}, \ldots\right\rangle$.

Let

$$
\mathfrak{M}=\left\langle H_{\theta}, \in,<,\left\langle\mathscr{C}_{\alpha}: \alpha \in \operatorname{Lim}(\lambda)\right\rangle, F, C,\left\{D_{n}: n \in \omega\right\}, \ldots\right\rangle .
$$

Take again a strictly $\subseteq$-increasing continuous sequence $\left\langle M_{\xi}: \xi<\lambda\right\rangle$ of elementary submodels of $\mathfrak{M}$ such that $\left|M_{\xi}\right|<\lambda$ and $M_{\xi} \cap \lambda$ is transitive for every $\xi<\lambda$. Then the set $\left\{M_{\xi} \cap \lambda: \xi<\lambda\right\}$ is a club in $\lambda$, and since $E_{\omega}^{\lambda}$ is stationary in $\lambda$, we can fix $M \preceq \mathfrak{M}$, with $M \cap \lambda$ transitive and $M \cap \lambda \in E_{\omega}^{\lambda}$. Let $\delta=M \cap \lambda$. We have the following:

Claim 4.2. There is an increasing sequence $\left\langle\delta_{n}: n<\omega\right\rangle$ of ordinals such that

(1) $\delta_{n} \in C \backslash \bigcup_{i \leq n} \operatorname{Lim}\left(C_{\delta}^{i}\right)$,

(2) $\sup \left\{\delta_{n}: n \in \omega\right\}=\delta$. 
Proof. Fix a strictly increasing sequence $\left\langle\epsilon_{n}: n\langle\omega\rangle \subseteq M\right.$ of limit $\delta$. We proceed by induction. To find $\delta_{0}$, apply directly Lemma 4.2 to find $\delta_{0} \in\left[\epsilon_{0}, \delta\right)$ with $\delta_{0} \in C \backslash \operatorname{Lim}\left(C_{\delta}^{0}\right)$. Fix $n \in \omega$, and suppose we have already built $\delta_{n}$ above $\epsilon_{n}$.

SUBCLAIM 4.1. There is a sequence of intervals $\left[\beta_{0}^{n}, \delta_{0}^{n}\right) \supseteq \cdots \supseteq\left[\beta_{i}^{n}, \delta_{i}^{n}\right)$ $\supseteq \cdots \supseteq\left[\beta_{n}^{n}, \delta_{n}^{n}\right)$ with $\beta_{0}^{n} \geq \max \left\{\delta_{n}, \epsilon_{n+1}\right\}$ and $\delta_{0}^{n}<\delta$, and there is a sequence $\left\langle M_{i}^{n} \preceq \mathfrak{M}_{n-i}: i \leq n\right\rangle$ of elementary submodels such that for every $i \leq n$,

- $\delta_{i}^{n}=\sup \left(M_{i}^{n} \cap \lambda\right)$,

- $\beta_{i}^{n} \in M_{i}^{n}$,

- $\left[\beta_{i}^{n}, \delta_{i}^{n}\right) \cap C_{\delta}^{j}=\emptyset$ for every $j \leq i$.

Proof. Since $D_{n} \in M$, apply Lemma 4.2 to $D_{n}, M, \operatorname{Lim}\left(C_{\delta}^{0}\right)$ and $\max \left\{\delta_{n}, \epsilon_{n+1}\right\}$ to find $\delta_{0}^{n}>\max \left\{\delta_{n}, \epsilon_{n+1}\right\}$ with $\delta_{0}^{n} \in D_{n} \cap M \backslash \operatorname{Lim}\left(C_{\delta}^{0}\right)$.

Let $M_{0}^{n} \preceq \mathfrak{M}_{n}$ be such that $\delta_{0}^{n}=\sup \left(M_{0}^{n} \cap \lambda\right)$. Take $\beta_{0}^{n} \in M_{0}^{n} \cap \lambda$ with $\beta_{0}^{n} \geq \max \left\{\delta_{n}, \epsilon_{n+1}\right\}$ and such that $\left[\beta_{0}^{n}, \delta_{0}^{n}\right) \cap C_{\delta}^{0}=\emptyset$.

Observe that for $n=0$ we are already done, so we can assume $n \geq 1$.

For $i<n$, suppose that we have found a sequence of intervals $\left[\beta_{0}^{n}, \delta_{0}^{n}\right) \supseteq$ $\cdots \supseteq\left[\beta_{i}^{n}, \delta_{i}^{n}\right)$ with $\beta_{0}^{n} \geq \max \left\{\delta_{n}, \epsilon_{n+1}\right\}$ and $\delta_{0}^{n}<\delta$ and a sequence $\left\langle M_{j}^{n} \preceq\right.$ $\left.\mathfrak{M}_{n-j}: j \leq i\right\rangle$ of elementary submodels such that for every $j \leq i$,

- $\delta_{j}^{n}=\sup \left(M_{j}^{n} \cap \lambda\right)$,

- $\beta_{j}^{n} \in M_{j}^{n}$,

- $\left[\beta_{j}^{n}, \delta_{j}^{n}\right) \cap C_{\delta}^{k}=\emptyset$ for every $k \leq j$.

Since $i<n$, the set $D_{n-i-1}$ is well-defined, and since $M_{i}^{n} \preceq \mathfrak{M}_{n-i}$, we have $D_{n-i-1} \in M_{i}^{n}$.

CASE 1: $\delta_{i}^{n} \notin \operatorname{Lim}\left(C_{\delta}^{i+1}\right)$. Choose $\beta_{i+1}^{n} \in M_{i}^{n} \cap \lambda$ with $\beta_{i+1}^{n} \geq \beta_{i}^{n}$ and such that $\left[\beta_{i+1}^{n}, \delta_{i}^{n}\right) \cap C_{\delta}^{i+1}=\emptyset$. Since $D_{n-i-1}$ is unbounded in $\lambda$, by elementarity we can find $\delta_{i+1}^{n} \in D_{n-i-1} \cap M_{i}^{n}$ with $\delta_{i+1}^{n}>\beta_{i+1}^{n}$, and thus $\left[\beta_{i+1}^{n}, \delta_{i+1}^{n}\right) \cap C_{\delta}^{j}=\emptyset$ for every $j \leq i+1$. Let $M_{i+1}^{n} \preceq M_{n-i-1}$ be such that $\delta_{i+1}^{n}=\sup \left(M_{i+1}^{n} \cap \lambda\right)$.

Case 2: $\delta_{i}^{n} \in \operatorname{Lim}\left(C_{\delta}^{i+1}\right)$. Take $k \in \omega$ such that $C_{\delta}^{i+1} \cap \delta_{i}^{n}=C_{\delta_{i}^{n}}^{k}$. Apply Lemma 4.2 to $M_{i}^{n}, \operatorname{Lim}\left(C_{\delta_{i}^{n}}^{k}\right), D_{n-i-1}$ and $\beta_{i}^{n}$ to find $\delta_{i+1}^{n} \in M_{i}^{n} \cap \lambda$ with $\delta_{i+1}^{n}>\beta_{i}^{n}$ and $\delta_{i+1}^{n} \notin \operatorname{Lim}\left(C_{\delta_{i}^{n}}^{k}\right)$. Let $M_{i+1}^{n} \preceq \mathfrak{M}_{n-i-1}$ be such that $\delta_{i+1}^{n}=$ $\sup \left(M_{i+1}^{n} \cap \lambda\right)$. Take $\beta_{i+1}^{n} \in M_{i+1}^{n}$ such that $\beta_{i+1}^{n} \geq \beta_{i}^{n}$ and $\left[\beta_{i+1}^{n}, \delta_{i+1}^{n}\right) \cap C_{\delta_{i}^{n}}^{k}$ $=\emptyset$. Then $\left[\beta_{i+1}^{n}, \delta_{i+1}^{n}\right) \cap C_{\delta}^{j}=\emptyset$ for every $j \leq i+1$.

Observe that we have defined $\beta_{n}^{n}, M_{n}^{n}$ and $\delta_{n}^{n}$ with $\delta_{n}^{n}=\sup \left(M_{n}^{n} \cap \lambda\right)$. To finish our construction, we again have two cases. 
CASE 1: $\delta_{n}^{n} \notin \operatorname{Lim}\left(C_{\delta}^{n+1}\right)$. Choose $\beta_{n+1}^{n} \in M_{n}^{n} \cap \lambda$ with $\beta_{n+1}^{n} \geq \beta_{n}^{n}$ and such that $\left[\beta_{n+1}^{n}, \delta_{n}^{n}\right) \cap C_{\delta}^{n+1}=\emptyset$. Since $C \in M_{n}^{n}$, by elementarity we can choose $\delta_{n+1} \in\left[\beta_{n+1}^{n}, \delta_{n}^{n}\right) \cap C$, and so $\delta_{n+1}$ is as needed.

CASE 2: $\delta_{n}^{n} \in \operatorname{Lim}\left(C_{\delta}^{n+1}\right)$. Take $k \in \omega$ such that $C_{\delta}^{n+1} \cap \delta_{n}^{n}=C_{\delta_{n}^{n}}^{k}$. Apply Lemma 4.2 to $M_{n}^{n}, \operatorname{Lim}\left(C_{\delta_{n}^{n}}^{k}\right), \beta_{n}^{n}$ and $C$ to find $\delta_{n+1} \in M_{n}^{n} \cap C \backslash \beta_{n}^{n}$ with $\delta_{n+1} \notin \operatorname{Lim}\left(C_{\delta_{n}^{n}}^{k}\right)$.

Now let $\beta_{n}<\delta_{n}$ such that $\left[\beta_{n}, \delta_{n}\right) \cap \bigcup_{i \leq n} C_{\delta}^{i}=\emptyset$. Then let $\left\langle\alpha_{n}, \gamma_{n}\right.$ : $n \in \omega\rangle$ be a sequence of I's moves according to $\tau$ against $\left\langle\beta_{n}: n \in \omega\right\rangle$. Moreover let $x=\operatorname{cl}_{F}\left(\left\{\gamma_{n}: n \in \omega\right\}\right)$. It suffices to prove that $x \in X$. To see this, first note that $\sup ^{+}(x)=\delta$ because $\delta$ is closed under $F$. We are going to check that setting $\xi_{n}^{x}=\delta_{n}$ will witness $x \in X$. Fix $n \in \omega$. Observe that for $m \geq n$, we have $C_{\delta}^{n} \cap \delta_{m} \subseteq \beta_{m} \subseteq \gamma_{m}$ by the choice of $\beta_{m}$. Also note that $\alpha_{m+1}<\delta_{m}$, because $\beta_{m} \in \delta_{m}$ and $\delta_{m}$ is closed under $\tau$ (since $\delta_{m} \in C$ ). Hence $C_{\delta}^{n} \cap\left[\delta_{m}, \delta_{m+1}\right) \subseteq\left[\alpha_{m+1}, \gamma_{m+1}\right)$ for every $m \geq n$. Note that $x \cap\left[\alpha_{k+1}, \gamma_{k+1}\right)=\emptyset$ for each $k \in \omega$ because I wins with the play $\left\langle\alpha_{k}, \beta_{k}, \gamma_{k}: k \in \omega\right\rangle$. Thus $x \cap C_{\delta}^{n} \subseteq \delta_{n}$. Moreover for $m \geq n$, $\min (x \backslash \beta)=\gamma_{m+1}$ for all $\beta \in C_{\delta}^{n} \cap\left[\delta_{m}, \delta_{m+1}\right)$, and $\operatorname{cof}\left(\gamma_{m+1}\right)=\omega_{1}$ by the rule of $G_{1}(\lambda, F)$. Therefore, $\delta_{n}=\xi_{n}^{x}$ witnesses $x \in X$.

This finishes the proof of Subclaim 4.1.

Claim 4.3. The hypothesis of Lemma 4.3 holds for $X$.

Proof. The proof is the same as in [19, proof of Claim 2], by fixing just one $C_{\delta}^{i}$ for some $i \in \omega$.

This completes the proof of Lemma 4.5.

5. Final remarks and open questions. Strong Chang's Conjecture is a consequence of the Weak Reflection Principle and Rado's Conjecture. Sakai and Veličković showed that WRP, together with $\mathrm{MA}_{\omega_{1}}$ (Cohen), implies that $\aleph_{2}$ has the Super Tree Property. However, they also showed that SSR and $\mathrm{MA}_{\omega_{1}}$ (Cohen) together do not imply $\omega_{2}$ has the Super Tree Property (see [19, Theorem 3.5]). Some natural questions arise: for $\omega_{2}$ ?

QUestion 5.1. Is WRP $+\neg \mathrm{CH}$ enough to have the Super Tree Property

Question 5.2. Does Rado's Conjecture, together with $\neg \mathrm{CH}$, imply $\omega_{2}$ has the Super Tree Property?

For example, it is known that if a strongly compact cardinal is Levy collapsed to $\omega_{2}$, then Rado's Conjecture holds. If starting from a model with a strongly compact cardinal $\kappa$, we can force Rado's Conjecture together with the negation of $\mathrm{CH}$ by a proper forcing which is an iteration of length $\kappa$ 
of small forcings, then this would answer this question negatively by [30, Corollary 6.10]. We thank the referee for pointing this out.

The following question is also still open:

QUESTION 5.3. Is $\mathrm{WRP}\left(\omega_{2}\right)$ enough to prove that the game $G\left(\omega^{2}\right)$ has a winning strategy, so we can get $\mathrm{WRP}\left(\omega_{2}\right)+\neg \mathrm{CH} \rightarrow \mathrm{TP}\left(\omega_{2}\right)$ ?

Acknowledgements. The first author was supported by Project $\mathrm{P}$ 26869-N25 of the Austrian Science Fund (FWF). The second author was supported by NSFC 11321101 and NSFC 11401567. He also wishes to thank Project P 26869-N25 of the Austrian Science Fund (FWF) for supporting a trip to Vienna.

\section{References}

[1] J. E. Baumgartner, Applications of the proper forcing axiom, in: Handbook of SetTheoretic Topology, North-Holland, Amsterdam, 1984, 913-959.

[2] J. Cummings and M. Magidor, Martin's maximum and weak square, Proc. Amer. Math. Soc. 139 (2011), 3339-3348.

[3] K. J. Devlin, The Yorkshireman's guide to proper forcing, in: Surveys in Set Theory, London Math. Soc. Lecture Note Ser. 87, Cambridge Univ. Press, Cambridge, 1983, 60-115.

[4] P. Doebler, Rado's conjecture implies that all stationary set preserving forcings are semiproper, J. Math. Logic 13 (2013), 1350001, 8 pp.

[5] P. Doebler and R. Schindler, $\Pi_{2}$ consequences of $\mathrm{BMM}+\mathrm{NS}_{\omega_{1}}$ is precipitous and the semiproperness of stationary set preserving forcings, Math. Res. Lett. 16 (2009), 797-815.

[6] P. Erdős and A. Tarski, On some problems involving inaccessible cardinals, in: Essays on the Foundations of Mathematics, Magnes Press, Hebrew Univ., Jerusalem, 1961, $50-82$.

[7] G. Fodor, Eine Bemerkung zur Theorie der regressiven Funktionen, Acta Sci. Math. (Szeged) 17 (1956), 139-142.

[8] M. Foreman, Ideals and generic elementary embeddings, in: Handbook of Set Theory. Vol. 2, Springer, Dordrecht, 2010, 885-1147.

[9] M. Foreman, M. Magidor, and S. Shelah, Martin's maximum, saturated ideals, and nonregular ultrafilters. I, Ann. of Math. (2) 127 (1988), 1-47.

[10] T. Jech, Set Theory, Springer Monogr. Math., Springer, Berlin, 2003.

[11] T. Jech, Stationary sets, in: Handbook of Set Theory. Vol. 1, Springer, Dordrecht, 2010, 93-128.

[12] T. J. Jech, Some combinatorial problems concerning uncountable cardinals, Ann. Math. Logic 5 (1972/73), 165-198.

[13] R. B. Jensen, The fine structure of the constructible hierarchy, Ann. Math. Logic 4 (1972), 229-308; Erratum, ibid. 4 (1972), 443.

[14] D. König, Über eine Schlussweise aus dem Endlichen ins Unendliche, Acta Sci. Math. (Szeged) 3 (1927), 121-130.

[15] G. Kurepa, Ensembles ordonnés et ramifiés, Publ. Math. Univ. Belgrade 4 (1935), 1-138. 
[16] W. Mitchell, Aronszajn trees and the independence of the transfer property, Ann. Math. Logic 5 (1972/73), 21-46.

[17] H. Sakai, Semistationary and stationary reflection, J. Symbolic Logic 73 (2008), 181-192.

[18] H. Sakai, Semi-stationary reflection and weak square, http://www2.kobe-u.ac.jp ${ }^{\sim} \sim$ hsakai/Research/notes/ssr_wsquare.pdf (2015).

[19] H. Sakai and B. Veličković, Stationary reflection principles and two cardinal tree properties, J. Inst. Math. Jussieu 14 (2015), 69-85.

[20] E. Schimmerling, Combinatorial principles in the core model for one Woodin cardinal, Ann. Pure Appl. Logic 74 (1995), 153-201.

[21] S. Shelah, Proper Forcing, Lecture Notes in Math. 940, Springer, Berlin, 1982.

[22] S. Shelah, Proper and Improper Forcing, 2nd ed., Perspect. Math. Logic, Springer, Berlin, 1998.

[23] R. Strullu, MRP, tree properties and square principles, J. Symbolic Logic 76 (2011), 1441-1452.

[24] S. Todorčević, A note on the proper forcing axiom, in: Axiomatic Set Theory (Boulder, CO, 1983), Contemp. Math. 31, Amer. Math. Soc., Providence, RI, 1984, 209-218.

[25] S. Todorčević, Conjectures of Rado and Chang and cardinal arithmetic, in: Finite and Infinite Combinatorics in Sets and Logic (Banff, AB, 1991), NATO Adv. Sci. Inst. Ser. C Math. Phys. Sci. 411, Kluwer, Dordrecht, 1993, 385-398.

[26] S. Todorčević and V. Torres-Pérez, Conjectures of Rado and Chang and special Aronszajn trees, Math. Logic Quart. 58 (2012), 342-347.

[27] S. Todorčević and V. Torres-Pérez, Rado's conjecture and ascent paths of square sequences, Math. Logic Quart. 60 (2014), no. 1-2, 84-90.

[28] V. Torres-Pérez and L. Wu, Strong Chang's conjecture and the tree property at $\omega_{2}$, Topology Appl. 196 (2015), 999-1004.

[29] B. Veličković, Forcing axioms and stationary sets, Adv. Math. 94 (1992), 256-284.

[30] M. Viale and C. Weiß, On the consistency strength of the proper forcing axiom, Adv. Math. 228 (2011), 2672-2687.

[31] C. Weiß, Subtle and ineffable tree properties, PhD thesis, 2010.

Víctor Torres-Pérez

Institut für Diskrete Mathematik und Geometrie

TU Wien

Wiedner Hauptstraße 8/104

1040 Wien, Austria

E-mail: victor.torres@tuwien.ac.at
Liuzhen $\mathrm{Wu}$

Institute of Mathematics Chinese Academy of Sciences East Zhong Guan Cun Road No. 55 Beijing 100190, China E-mail: lzwu@math.ac.cn 